\title{
On a Generalization of Hofstadter's Q-Sequence: A Family of Chaotic Generational Structures
}

\author{
Altug Alkan (i) \\ Graduate School of Science and Engineering, Piri Reis University, 34940 Tuzla, Istanbul, Turkey \\ Correspondence should be addressed to Altug Alkan; altug.alkan1988@gmail.com
}

Received 27 November 2017; Accepted 2 May 2018; Published 25 June 2018

Academic Editor: Dimitri Volchenkov

Copyright ( 2018 Altug Alkan. This is an open access article distributed under the Creative Commons Attribution License, which permits unrestricted use, distribution, and reproduction in any medium, provided the original work is properly cited.

\begin{abstract}
Hofstadter $Q$-recurrence is defined by the nested recurrence $Q(n)=Q(n-Q(n-1))+Q(n-Q(n-2))$, and there are still many unanswered questions about certain solutions of it. In this paper, a generalization of Hofstadter's $Q$-sequence is proposed and selected members of this generalization are investigated based on their chaotic generational structures and Pinn's statistical technique. Solutions studied have also curious approximate patterns and considerably similar statistical properties with Hofstadter's famous Q-sequence in terms of growth characteristics of their successive generations. In fact, the family of sequences that this paper introduces suggests the existence of conjectural global properties in order to classify unpredictable solutions to Q-recurrence and a generalization of it.
\end{abstract}

\section{Introduction}

Since the enigmatic concept of meta-Fibonacci has been introduced by Douglas Hofstadter with the invention of the original Q-sequence (A005185 in OEIS), in the literature, there are many studies which focus on nested recurrence relations whose behaviors can alternate dramatically [1-5]. There are many examples of meta-Fibonacci sequences like Hofstadter-Conway \$10000 sequence (A004001), Conolly sequence (A046699), Tanny sequence (A006949), Golomb's sequence (A001462), Mallows' sequence (A005229), etc. [6-10]. Some of meta-Fibonacci sequences are highly chaotic and unpredictable while some of them have completely predictable fashion such as quasipolynomial solutions to the Hofstadter Q-recurrence [11-13] and slow $V$-sequence (A063882) that is also 2-automatic [14]. Among the solutions which have an erratic nature, certain variants have underlying structures that contain conjecturally interesting approximate properties such as scaling, self-similarity, and period doubling $[15,16]$. For these kinds of solutions of nested recurrences, known mathematical techniques for solving difference equations do not work because of the nature of nesting although there are alternative definitions for the generational structure of a chaotic meta-Fibonacci sequence [15-19]. The existence of universality classes for chaotic meta-Fibonacci sequences determined by common characteristics of their respective generational structures is a mysterious open question although there are a variety of attempts in order to search an affirmative answer for this question, partially [15-17]. Since the certain solutions to the Hofstadter Q-recurrence are investigated in this study, it would be nice to remember the scatterplots of Hofstadter's original Q-sequence and the "Brother" sequence (A284644) that is defined by $Q_{b}(n)=$ $Q_{b}\left(n-Q_{b}(n-1)\right)+Q_{b}\left(n-Q_{b}(n-2)\right)$ and initial values $Q_{b}$ $(1)=Q_{b}(2)=2$ and $Q_{b}(3)=1$ (see Figure 1). Although there are many solutions to the Hofstadter Q-recurrence with different initial conditions $[9,11-13,17,20]$, these two solutions and their connection based on their generational structures provide a variety of interesting experimental results [17].

This paper is structured as follows. In Section 2, Hofstadter's Q-sequence is generalized according to the initial condition formulation and an intriguing sequence family is introduced. Then, in Section 3.1 and Section 3.2, selected members of this curious sequence family are studied based on their generational structures with the statistical perspective. Finally, some concluding remarks are offered in Section 4. 

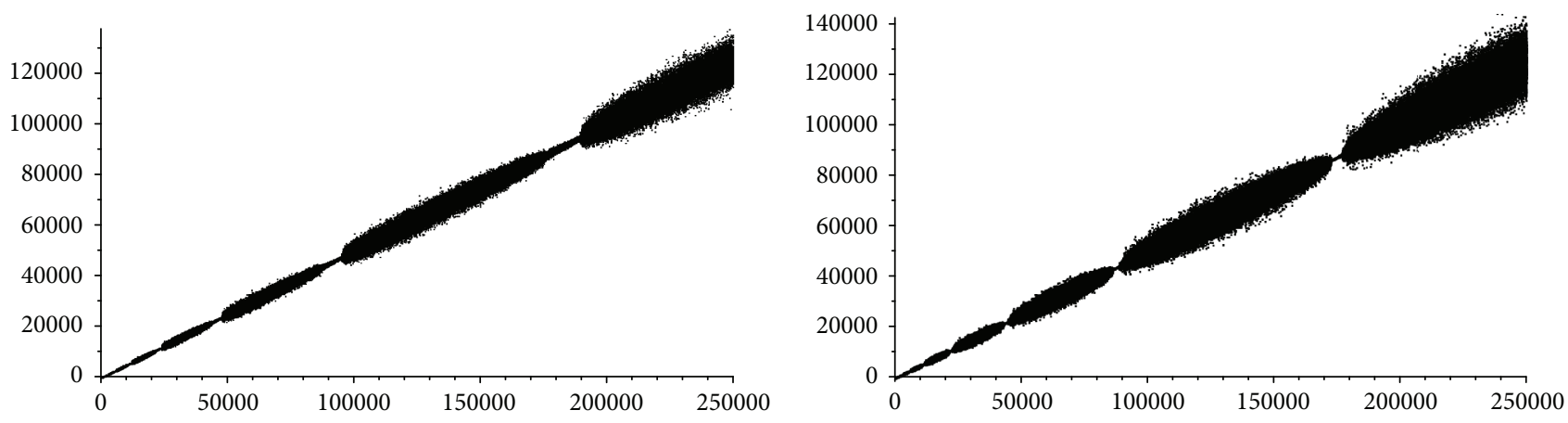

Figure 1: Scatterplots of Hofstadter's $Q$-sequence and $Q_{b}(n)$.

\section{A Generalization of Hofstadter's Q-Sequence according to Initial Conditions}

Hofstadter's $Q$-sequence is a very intriguing solution to the $Q$

-recurrence, and it is believed that the most notable metaFibonacci sequence is Hofstadter's Q-sequence [11]. Indeed, many famous mathematicians such as Erdös, Guy, and Sloane found it really very interesting $[21,22]$. At this point, it is natural to ask, "Can a generalization of solutions to the $Q$-recurrence be based on the initial conditions for variants that behave in a similar fashion with a $Q$-sequence?" If the answer is yes, there can be a collection of curious chaotic patterns and generational structures hidden in genes of the $Q$-recurrence. Solutions with three initial conditions are studied before [17]. In order to go further, computer experiments can be made with four and five initial conditions but empirical results suggest that there is no sign of any sequence family with generational common characteristics except Q-sequence and "Brother" sequence (A284644) although there are some more chaotic solutions such as A278056 [9, 11]. So experiments suggest that computation of all living permutations of initial conditions is not very fruitful in order to discover a solution family that has members which behave very similar with the original $Q$-sequence. Also, recently, the Hofstadter Q-recurrence and a generalization of it are studied with initial conditions 1 through $N$ and detailed analysis showed that living solutions have notably different properties from the $Q$-sequence with the increasing values of $N$ [11]. At this point, it would be nice to remember if $\lim _{n \rightarrow \infty} Q(n) / n$ exists, it must be equal to $1 / 2$ [13]. From this fact, initial conditions which are $\lceil n / 2\rceil$ may be meaningful for the Hofstadter Q-recurrence. Additionally, this approach inspired by reasonable heuristic can be generalized with the initial condition formulation as below.

Definition 1. Let $Q_{d, l}(n)$ be defined by the recurrence $Q_{d, l}$ $(n)=\sum_{i=1}^{l} Q_{d, l}\left(n-Q_{d, l}(n-i)\right)$ for $n>d * l, l \geq 2$, and $d \geq 1$, with the initial conditions $Q_{d, l}(n)=\left\lceil n^{*}(l-1) / l\right\rceil$ for $n \leq d * l$.

By definition, $Q_{1,2}$ is the original $Q$-sequence and $Q_{3,2}$ is essentially the same with $Q_{1,2} \cdot Q_{2,2}$ is extremely wild sequence that there are no signs of any underlying structure (see Figure 2).

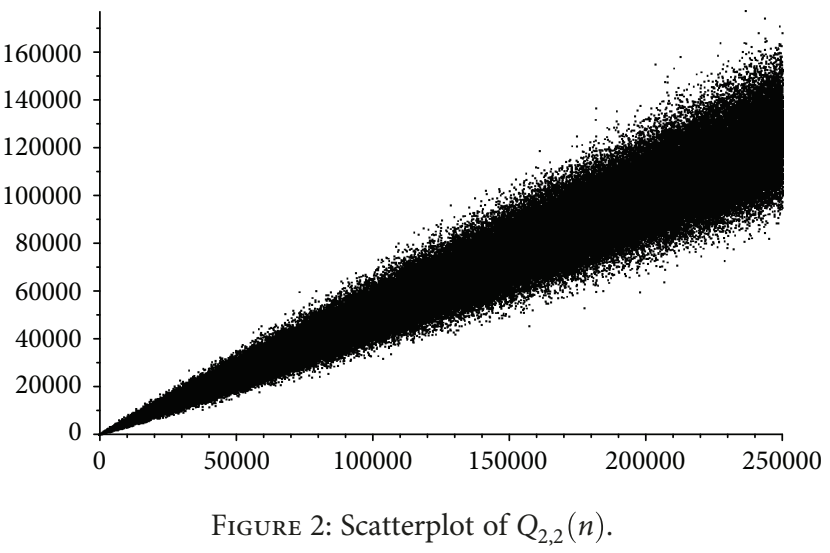

However, for $d \geq 4$, many curious chaotic patterns fascinatingly start to appear for $S_{d, 2}(n)=Q_{d, 2}(n)-n / 2$ (see Figure 3 ). In the next section, certain members of this family are investigated in order to search the signs of a similar inheritance with the original $Q$-sequence although $Q_{2,2}$ exhibits quite different experimental characteristics, at least in the range of this study.

In this study, solutions to $Q_{d, 2}(n)$ and $Q_{d, 3}(n)$ recurrences will be studied with certain examples while $Q_{d, l}(n)$ continues to provide intriguing generational structures with an increasing level of complexity for $l \geq 4$.

\section{Analysis of Certain Members of $Q_{d, l}(n)$ Family}

3.1. Selected Solutions to $Q_{d, 2}(n)$. Approximate self-similar block structures of certain members of the $Q_{d, 2}(n)$ family can be studied thanks to auxiliary sequences similar with different works which give definitions of generations [15-18]. In here, the main purpose is to model and compute the rescaling of amplitudes for self-similar successive block structures of $S_{d, 2}(n)$ for the selected values of $d$ which are in the range of this study, since this computation will give a chance to search a conjectural global property for certain solutions to the Hofstadter Q-recurrence. Certain auxiliary sequences can be used in order to compute statistical quantities which this paper focuses on. Experiments that use alternative definitions for the determination of generational boundaries are also carried out precisely. Since the results are mainly similar in terms of the values of Table 1 , only 

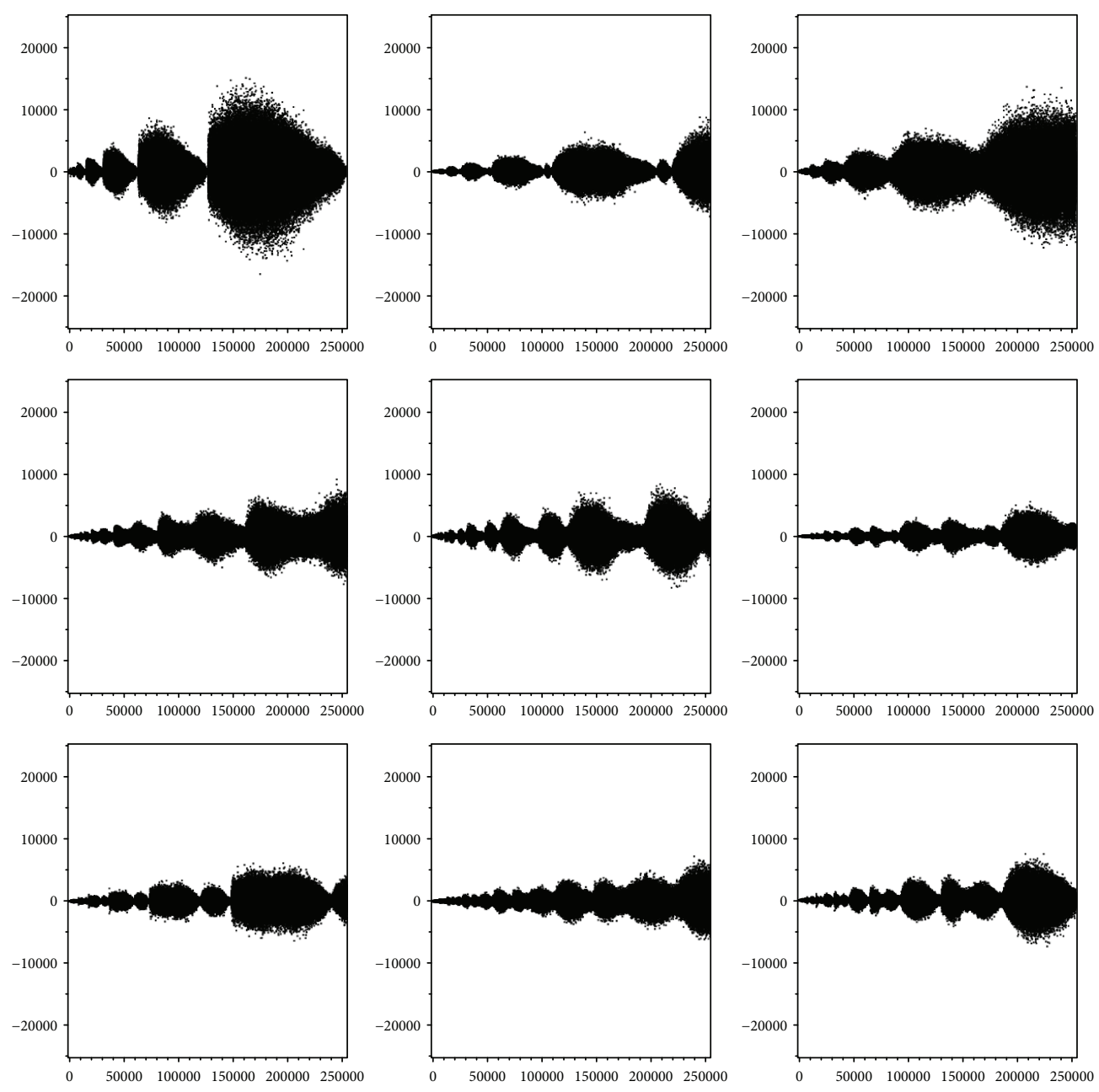

Figure 3: Scatterplots of $S_{d, 2}(n)$ for $4 \leq d \leq 12$, respectively.

TABLE 1: Values of $\alpha\left(k, S_{4,2}(n)\right), \alpha\left(k, S_{7,2}(n)\right)$, and $\alpha\left(k, S_{10,2}(n)\right)$ for $10 \leq k \leq 25$.

\begin{tabular}{lccc}
\hline$k$ & $\alpha\left(k, S_{4,2}(n)\right)$ & $\alpha\left(k, S_{7,2}(n)\right)$ & $\alpha\left(k, S_{10,2}(n)\right)$ \\
\hline 10 & 0.847 & 0.798 & 0.837 \\
11 & 0.828 & 0.895 & 0.776 \\
12 & 0.853 & 0.886 & 0.803 \\
13 & 0.764 & 0.824 & 0.883 \\
14 & 0.869 & 0.861 & 0.877 \\
15 & 0.858 & 0.875 & 0.880 \\
16 & 0.862 & 0.870 & 0.863 \\
17 & 0.875 & 0.890 & 0.877 \\
18 & 0.869 & 0.880 & 0.886 \\
19 & 0.878 & 0.884 & 0.882 \\
20 & 0.884 & 0.883 & 0.882 \\
21 & 0.882 & 0.886 & 0.884 \\
22 & 0.883 & 0.885 & 0.885 \\
23 & 0.885 & 0.884 & 0.886 \\
24 & 0.887 & 0.886 & 0.886 \\
25 & 0.888 & 0.886 & 0.886 \\
\hline
\end{tabular}

one method's table is reported in here. Corresponding method's definitions follow as below. See Figure 4 for some examples of partitions.

Definition 2. Let $W_{d, 2}(n)$ be the least $m$ such that the minimum of father $\left(m-Q_{d, 2}(m-2)\right)$ and mother $\left(m-Q_{d, 2}(m-1)\right)$ spots is equal or greater than $n$.

Definition 3. Let $P_{d, 2}(n)=W_{d, 2}\left(P_{d, 2}(n-1)\right)$ where $d \in\{4,7$, $10\}$ for $n>1$, with $P_{d, 2}(1)=1$.

See Tables 2-4 for the corresponding values of $P_{d, 2}(n)$.

The given sequence $S_{d, 2}(n)=Q_{d, 2}(n)-n / 2,\left\langle S_{d, 2}(n)\right\rangle_{k}$ denotes the average value of $S_{d, 2}(n)$ over the $k$ th generation boundaries that are determined by $P_{d, 2}(n)$ for corresponding $Q_{d, 2}(n)$ and define $\alpha\left(k, S_{d, 2}(n)\right)$ as below. See Table 1 and Figure 5 in order to observe the considerable similarities between $\alpha$ values with the increasing number of generations. These results are very close to the values that are reported before [15-17]. In other words, the initial condition pattern that this study focuses on provides significant behavioral similarities with the original $Q$-sequence in 

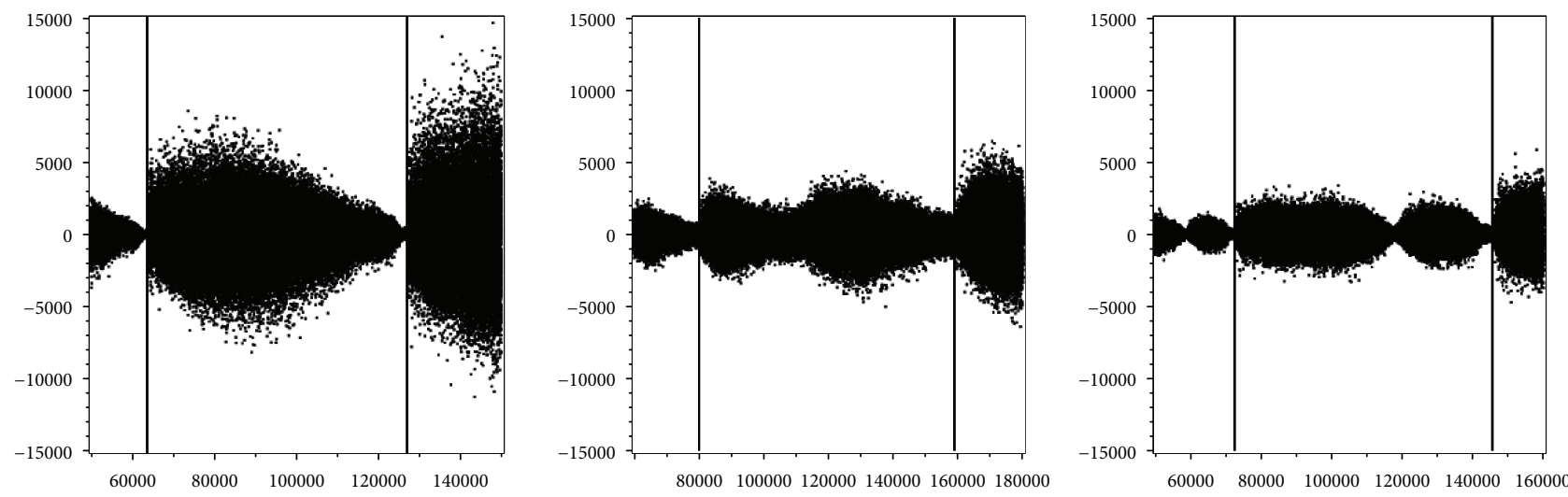

FIGURE 4: Illustrations of $P_{d, 2}(17)$ and $P_{d, 2}(18)$ on scatterplots of $S_{d, 2}(n)$ where $d \in\{4,7,10\}$, respectively.

TABLE 2: The values of $P_{4,2}(n)$ sequence for $n \leq 25$.

\begin{tabular}{lccccc}
\hline & 1 & 2 & $m$ & 3 & 5 \\
\hline$P_{4,2}(\mathrm{~m}+0)$ & 1 & 3 & 5 & 9 & 17 \\
$P_{4,2}(\mathrm{~m}+5)$ & 33 & 65 & 129 & 257 & 513 \\
$P_{4,2}(\mathrm{~m}+10)$ & 1025 & 2049 & 4088 & 8163 & 16227 \\
$P_{4,2}(\mathrm{~m}+15)$ & 32206 & 63943 & 127182 & 253527 & 704715 \\
$P_{4,2}(\mathrm{~m}+20)$ & 1001529 & 1990206 & 3956008 & 7852309 & 15566939 \\
\hline
\end{tabular}

TABLE 3: The values of $P_{7,2}(n)$ sequence for $n \leq 25$.

\begin{tabular}{lccccc}
\hline & 1 & 2 & $m$ & 3 & 9 \\
\hline$P_{7,2}(\mathrm{~m}+0)$ & 1 & 3 & 5 & 3 & 9 \\
$P_{7,2}(\mathrm{~m}+5)$ & 37 & 76 & 155 & 314 & 630 \\
$P_{7,2}(\mathrm{~m}+10)$ & 1264 & 2538 & 5076 & 10155 & 20269 \\
$P_{7,2}(\mathrm{~m}+15)$ & 40309 & 80178 & 158920 & 315670 & 626261 \\
$P_{7,2}(\mathrm{~m}+20)$ & 1242680 & 2461343 & 4881527 & 9689364 & 19208568 \\
\hline
\end{tabular}

TABLE 4: The values of $P_{10,2}(n)$ sequence for $n \leq 25$.

\begin{tabular}{|c|c|c|c|c|c|}
\hline & \multicolumn{5}{|c|}{$m$} \\
\hline & 1 & 2 & 3 & 4 & 5 \\
\hline$P_{10,2}(m+0)$ & 1 & 3 & 5 & 9 & 17 \\
\hline$P_{10,2}(\mathrm{~m}+5)$ & 34 & 69 & 140 & 283 & 569 \\
\hline$P_{10,2}(\mathrm{~m}+10)$ & 1141 & 2285 & 4573 & 9147 & 18292 \\
\hline$P_{10,2}(\mathrm{~m}+15)$ & 36542 & 72974 & 145867 & 291183 & 581442 \\
\hline$P_{10,2}(\mathrm{~m}+20)$ & 1160383 & 2313867 & 4614469 & 9202451 & 18337568 \\
\hline
\end{tabular}




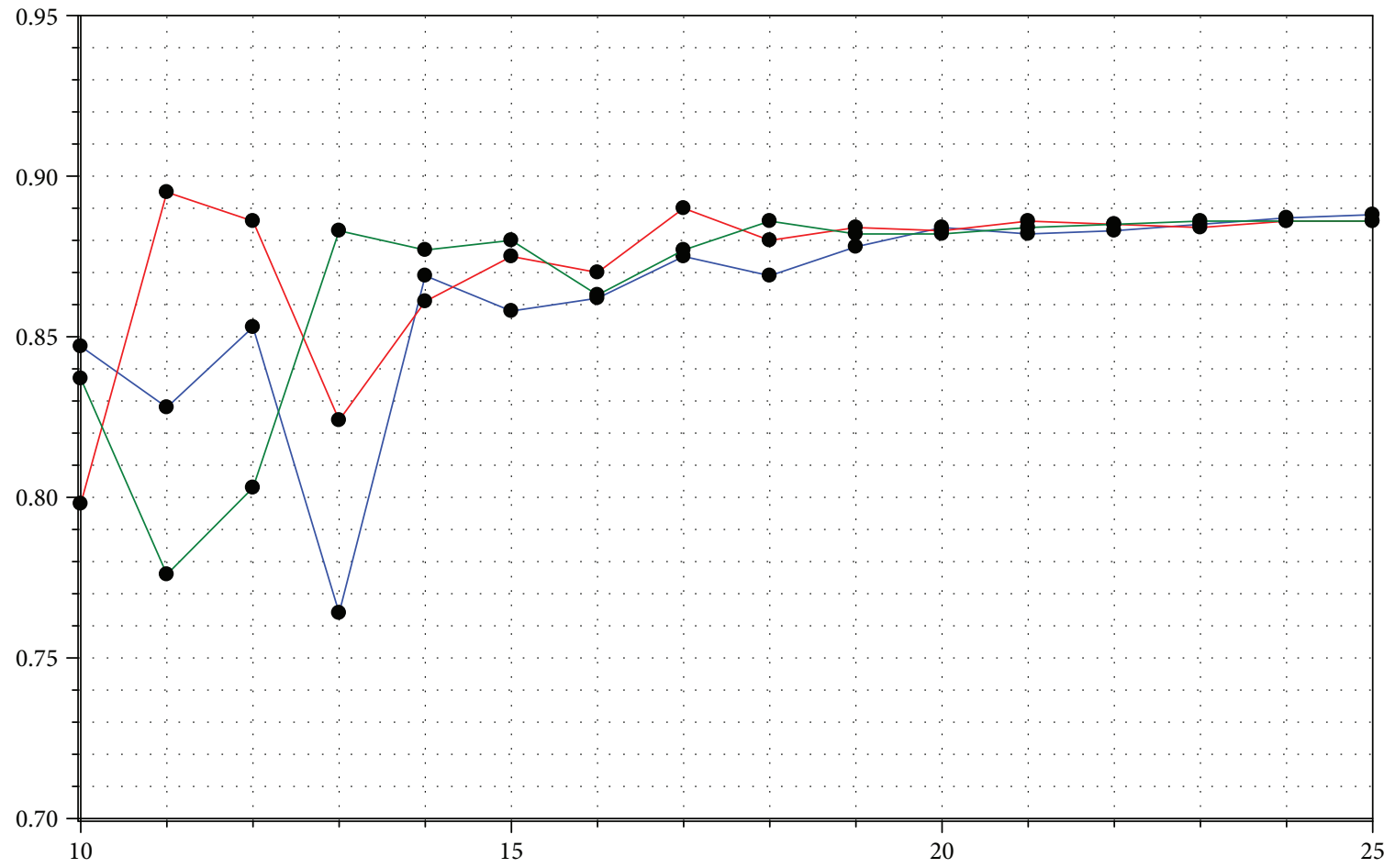

Figure 5: Blue: $\alpha\left(k, S_{4,2}(n)\right)$. Red: $\alpha\left(k, S_{7,2}(n)\right)$. Green: $\alpha\left(k, S_{10,2}(n)\right)$.

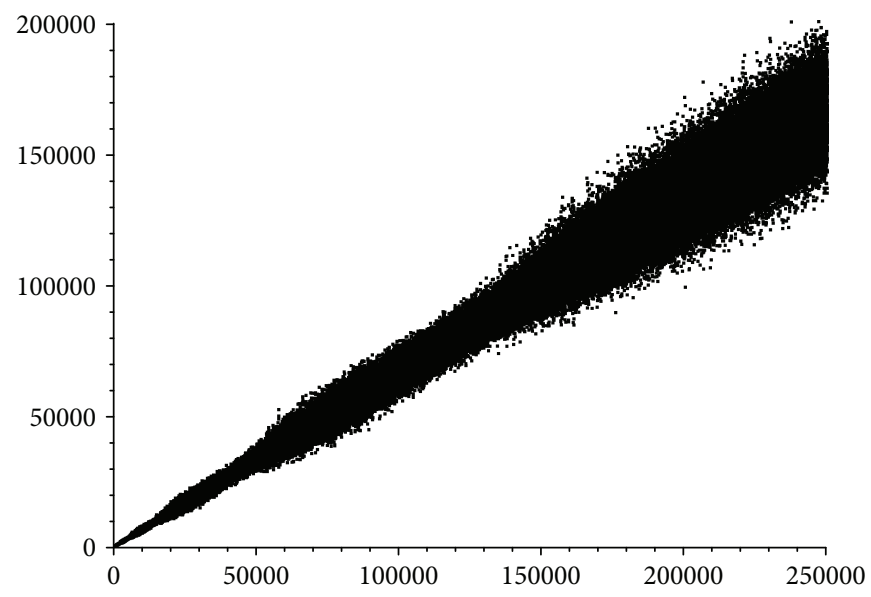

Figure 6: Scatterplot of $Q_{3,3}(n)$.
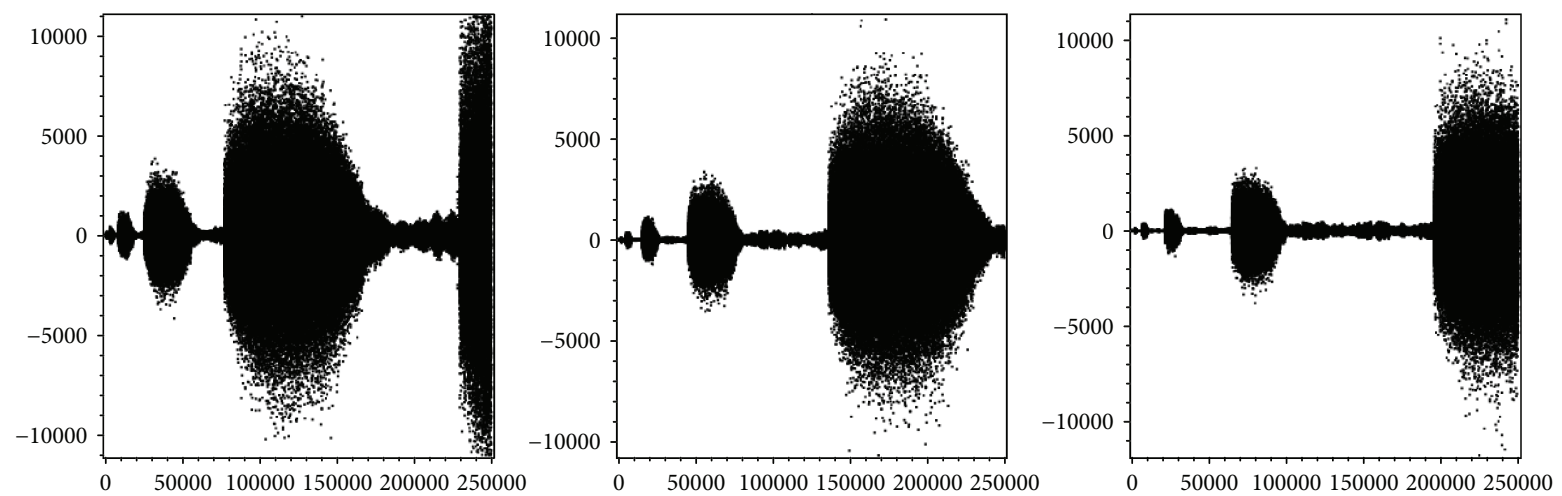

FIGURE 7: Scatterplots of $S_{d, 3}(n)$ where $d \in\{4,7,10\}$, respectively. 
TABLE 5: The values of $g_{4,3}(n)$ sequence for $n \leq 15$.

\begin{tabular}{lccccc}
\hline & 1 & 2 & $m$ & 4 & 5 \\
\hline$g_{4,3}(m+0)$ & 1 & 13 & 39 & 114 & 327 \\
$g_{4,3}(m+5)$ & 970 & 2911 & 8650 & 25875 & 77058 \\
$g_{4,3}(m+10)$ & 228424 & 678683 & 2020707 & 6016683 & 17966896 \\
\hline
\end{tabular}

TABLE 6: The values of $g_{7,3}(n)$ sequence for $n \leq 15$.

\begin{tabular}{lccccc}
\hline & 1 & 2 & 3 & 4 & 5 \\
\hline$g_{7,3}(m+0)$ & 1 & 22 & 66 & 195 & 570 \\
$g_{7,3}(m+5)$ & 1699 & 5102 & 15224 & 45510 & 136182 \\
$g_{7,3}(m+10)$ & 406324 & 1209535 & 3611564 & 10797842 & 32259345 \\
\hline
\end{tabular}

TABLE 7: The values of $g_{10,3}(n)$ sequence for $n \leq 15$.

\begin{tabular}{lccccc}
\hline & 1 & 2 & 3 & 4 & 5 \\
\hline$g_{10,3}(m+0)$ & 1 & 31 & 93 & 276 & 813 \\
$g_{10,3}(m+5)$ & 2428 & 7289 & 21802 & 65263 & 195493 \\
$g_{10,3}(m+10)$ & 584332 & 1743893 & 5216310 & 15587996 & 46668176 \\
\hline
\end{tabular}
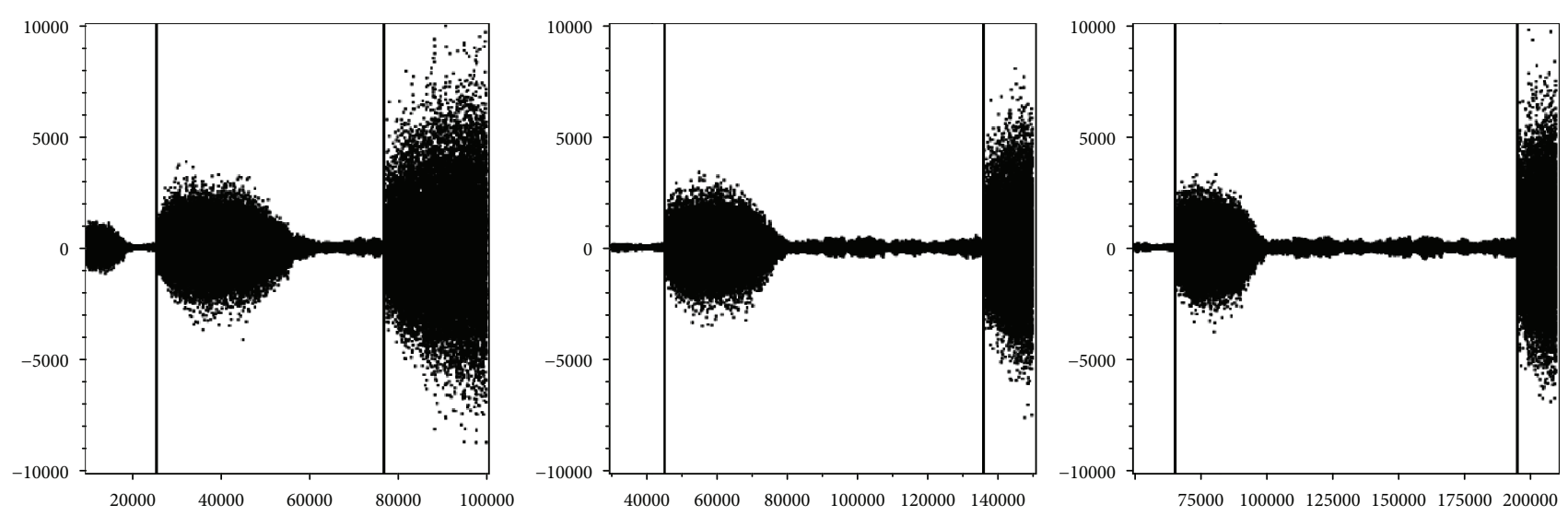

FIGURE 8: Illustrations of $g_{d, 3}(9)$ and $g_{d, 3}(10)$ on scatterplots of $S_{d, 3}(n)$ where $d \in\{4,7,10\}$, respectively.

terms of growth characteristics of successive generations. Additionally, other solutions for $4 \leq d \leq 12$ are checked thanks to certain auxiliary sequences and careful examination of their data. Results observed are mainly similar to the values of Table 1 .

$$
\begin{aligned}
M_{k}\left(S_{d, 2}(n)\right)^{2} & =\left\langle S_{d, 2}(n)^{2}\right\rangle_{k}-\left\langle S_{d, 2}(n)\right\rangle_{k}^{2}, \\
\alpha\left(k, S_{d, 2}(n)\right) & =\log _{2}\left(\frac{M_{k}\left(S_{d, 2}(n)\right)}{M_{k-1}\left(S_{d, 2}(n)\right)}\right) .
\end{aligned}
$$

3.2. Selected Solutions to $Q_{d, 3}(n)$. In this section, certain members of $Q_{d, 3}(n)$ are analysed thanks to properties of their generational structures. It is easy to show that $Q_{1,3}(n)$ and $Q_{2,3}(n)$ die immediately since $Q_{1,3}(4)=6$ and $Q_{2,3}(66)=73$. See Figure 6 for $Q_{3,3}(n)$ that is highly chaotic sequence although there appear to be some weak signs of order in it. Then, more orderly generational structures evolve in terms of the determination of main blocks (see Figure 7 for curious examples where $S_{d, 3}(n)=$ $\left.Q_{d, 3}(n)-2 * n / 3\right)$. In that case, in order to detect the limits 
TABLE 8: Values of $\alpha\left(k, S_{4,3}(n)\right), \alpha\left(k, S_{7,3}(n)\right)$, and $\alpha\left(k, S_{10,3}(n)\right)$ for $5 \leq k \leq 15$.

\begin{tabular}{lccc}
\hline$k$ & $\alpha\left(k, S_{4,3}(n)\right)$ & $\alpha\left(k, S_{7,3}(n)\right)$ & $\alpha\left(k, S_{10,3}(n)\right)$ \\
\hline 5 & 0.837 & 0.838 & 0.838 \\
6 & 0.937 & 0.925 & 0.926 \\
7 & 0.928 & 0.894 & 0.898 \\
8 & 0.936 & 0.927 & 0.933 \\
9 & 0.937 & 0.944 & 0.942 \\
10 & 0.954 & 0.947 & 0.949 \\
11 & 0.951 & 0.952 & 0.949 \\
12 & 0.948 & 0.951 & 0.949 \\
13 & 0.952 & 0.952 & 0.953 \\
14 & 0.952 & 0.954 & 0.953 \\
15 & 0.954 & 0.954 & 0.954 \\
\hline
\end{tabular}

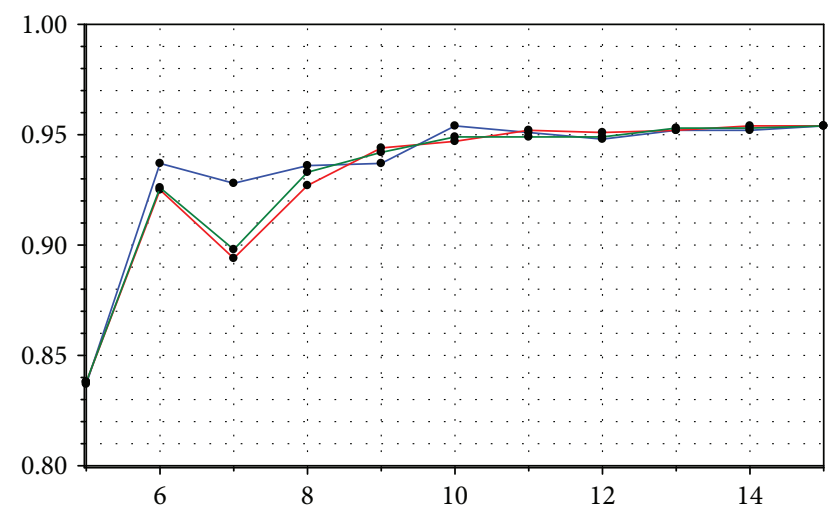

Figure 9: Blue: $\alpha\left(k, S_{4,3}(n)\right)$. Red: $\alpha\left(k, S_{7,3}(n)\right)$. Green: $\alpha\left(k, S_{10,3}(n)\right)$.

of approximate self-similar block structures, spot-based generation concept can be used for $d \in\{4,7,10\}$ based on spot $n-Q_{d, 3}(n-1)$ [18].

Definition 4. Let $g_{d, 3}(n)$ be the least value of $t$ such that $M_{p, d, 3}(t)$ is equal to $n$ where $d \in\{4,7,10\}$ and $M_{p, d, 3}(n)=$ $M_{p, d, 3}\left(n-Q_{d, 3}(n-1)\right)+1$ with initial conditions $M_{p, d, 3}(n)=$ 1 for $n \leq 3 * d$.

See Tables 5-7 for the corresponding values of $g_{d, 3}(n)$ and Figure 8 for some illustrations of generational boundaries.

Similar with the previous section, for a given sequence $S_{d, 3}(n)=Q_{d, 3}(n)-2 * n / 3,\left\langle S_{d, 3}(n)\right\rangle_{k}$ denotes the average value of $S_{d, 3}(n)$ over the $k$ th generation boundaries that are determined by $g_{d, 3}(n)$ for corresponding $Q_{d, 3}(n)$ and define $\alpha\left(k, S_{d, 3}(n)\right)$ as below. Also, similarly, see Table 8 and Figure 9 in order to observe the considerable similarities between $\alpha$ values that are different from the values which are reported in the previous section since the recurrence is $Q(n)=Q(n-Q(n-1))+Q(n-Q(n-2))+Q(n-Q(n-3))$ in that case.

$$
\begin{aligned}
M_{k}\left(S_{d, 3}(n)\right)^{2} & =\left\langle S_{d, 3}(n)^{2}\right\rangle_{k}-\left\langle S_{d, 3}(n)\right\rangle_{k}^{2}, \\
\alpha\left(k, S_{d, 3}(n)\right) & =\log _{3}\left(\frac{M_{k}\left(S_{d, 3}(n)\right)}{M_{k-1}\left(S_{d, 3}(n)\right)}\right) .
\end{aligned}
$$

\section{Conclusion}

In the literature, there are many studies that are primarily concerned with finding initial conditions to corresponding meta-Fibonacci recurrences where the solutions have a provable universal property such as having an ordinary generating function and being slow $[11,12,20]$. On the other hand, properties of Hofstadter's Q-sequence depend on experimental studies because of its complicated nature that is extremely resistant to known mathematical proof techniques $[15-17,19]$. In this study, a variety of evidences are provided in order to claim the existence of a family that certain members have considerably similar conjectural properties with famous $Q$-sequence. A generalization of $Q$-sequence according to the initial condition patterns which are determined by asymptotic properties of recurrences is introduced, and meaningful statistical results are provided in terms of the classification of chaotic solutions to recurrences that this study focuses on.

\section{Conflicts of Interest}

The author declares that there is no conflict of interest regarding the publication of this paper.

\section{Acknowledgments}

The author would like to thank D.R. Hofstadter for a valuable feedback about some sequences which are fascinating mixtures of regularity and irregularity. The author would also like to thank Robert Israel regarding his valuable help for Maple-related requirements of this study.

\section{References}

[1] B. V. S. Bharadwaj, L. S. Chandran, and A. Das, "Isoperimetric problem and meta-fibonacci sequences," in Computing and Combinatorics. COCOON 2008, X. Hu and J. Wang, Eds., vol. 5092 of Lecture Notes in Computer Science, Springer, Berlin, Heidelberg, 2008.

[2] D. Hofstadter, "Godel, escher, bach: an eternal golden braid," in Basic Books, New York, NY, USA, 1979.

[3] D. Hofstadter, "Curious patterns and nonpatterns in a family of metafibonacci recursions," in talk given at Rutgers Experimental Mathematics Seminar, Camden, NJ, USA, 2014, https:// vimeo.com/91708646.

[4] A. Isgur, D. Reiss, and S. Tanny, "Trees and meta-Fibonacci sequences," Electronic Journal of. Combinatorics, vol. 16, article R129, p. 1, 2009.

[5] A. Isgur, R. Lech, S. Moore, S. Tanny, Y. Verberne, and Y. Zhang, "Constructing new families of nested recursions with slow solutions," SIAM Journal on Discrete Mathematics, vol. 30, no. 2, pp. 1128-1147, 2016.

[6] B. W. Conolly, "Meta-Fibonacci sequences," in Fibonacci and Lucas Numbers, and the Golden Section, pp. 127-138, Wiley, 1989. 
[7] T. Kubo and R. Vakil, "On Conway's recursive sequence," Discrete Mathematics, vol. 152, no. 1-3, pp. 225-252, 1996.

[8] C. L. Mallows, "Conway's challenge sequence," The American Mathematical Monthly, vol. 98, no. 1, pp. 5-20, 1991.

[9] N. J. A. Sloane, OEIS Foundation Inc, The On-Line Encyclopedia of Integer Sequences, 2018.

[10] S. M. Tanny, "A well-behaved cousin of the Hofstadter sequence," Discrete Mathematics, vol. 105, no. 1-3, pp. 227239, 1992

[11] N. Fox, An Exploration of Nested Recurrences Using Experimental Mathematics, [Ph.D. thesis], Rutgers The State University of New Jersey-New Brunswick, 2017.

[12] N. Fox, Quasipolynomial Solutions to the Hofstadter Q-Recurrence, INTEGERS, 2016.

[13] S. W. Golomb, Discrete chaos: sequences satisfying strange recursions, 1991.

[14] J. P. Allouche and J. Shallit, “A variant of Hofstadter's sequence and finite automata," 2011, http://arxiv.org/abs/1103.1133.

[15] K. Pinn, "A chaotic cousin of Conway's recursive sequence," Experimental Mathematics, vol. 9, no. 1, pp. 55-66, 2000.

[16] K. Pinn, "Order and chaos in Hofstadter's $Q(n)$ sequence," Complexity, vol. 4, no. 3, 46 pages, 1999.

[17] A. Alkan, N. Fox, and O. Ozgur Aybar, "On Hofstadter heart sequences," Complexity, vol. 2017, Article ID 2614163, 8 pages, 2017.

[18] B. Dalton, M. Rahman, and S. Tanny, "Spot-based generations for meta-Fibonacci sequences," Experimental Mathematics, vol. 20, no. 2, pp. 129-137, 2011.

[19] S. M. Tanny, "An invitation to nested recurrence relations," in 4th Biennial Canadian Discrete and Algorithmic Mathematics Conference (CanaDAM), pp. 6-7, Canada, 2013, https:// canadam.math.ca/2013/program/slides/Tanny.Steve.pdf.

[20] F. Ruskey, "Fibonacci meets Hofstadter," Fibonacci Quart, vol. 49, no. 3, pp. 227-230, 2011.

[21] R. K. Guy, Unsolved Problems in Number Theory, Problem E31, Springer-Verlag, 1994.

[22] D. Hofstadter and N. J. A. Sloane, Correspondence1977 and 1991, https://oeis.org/A006336/a006336.pdf. 


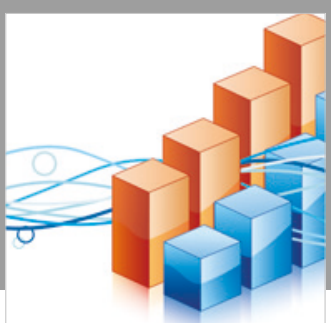

Advances in

Operations Research

\section{-n-m}
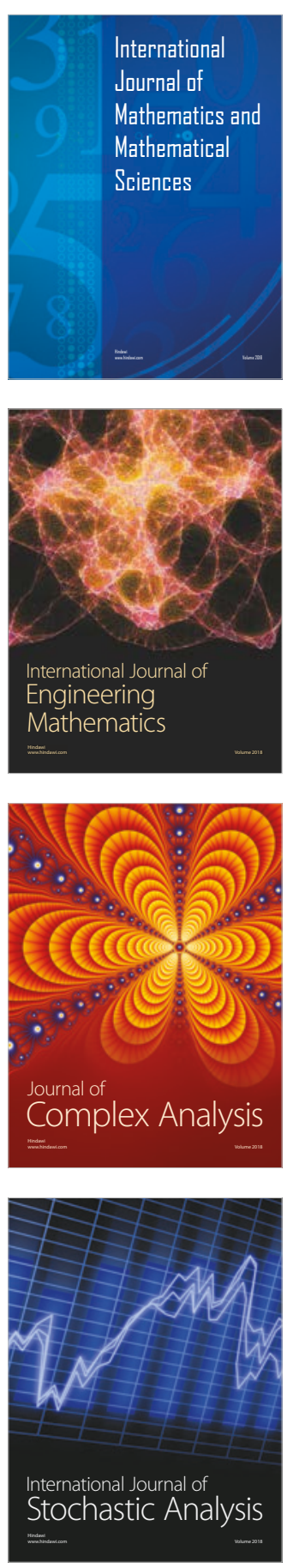
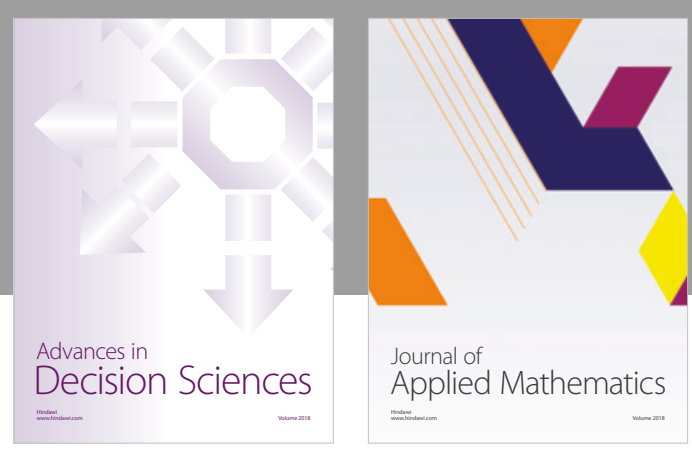

Journal of

Applied Mathematics
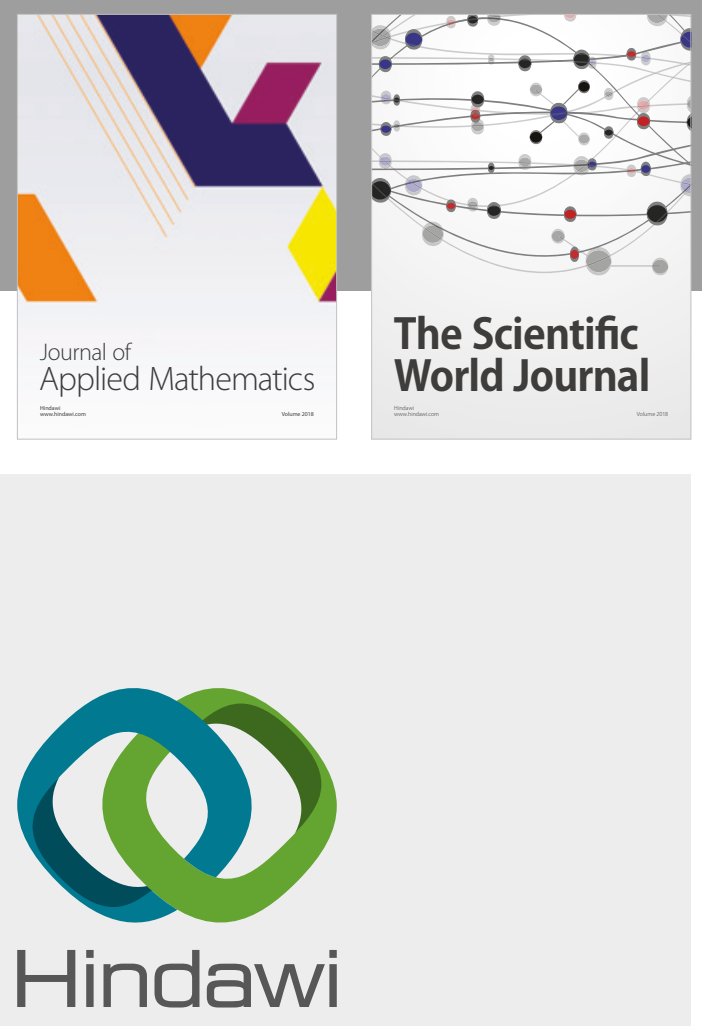

Submit your manuscripts at

www.hindawi.com

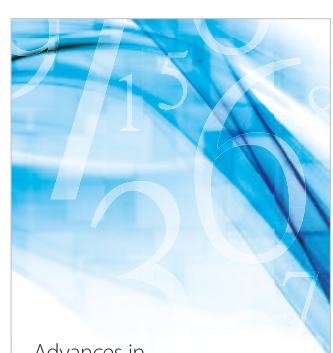

Advances in
Numerical Analysis
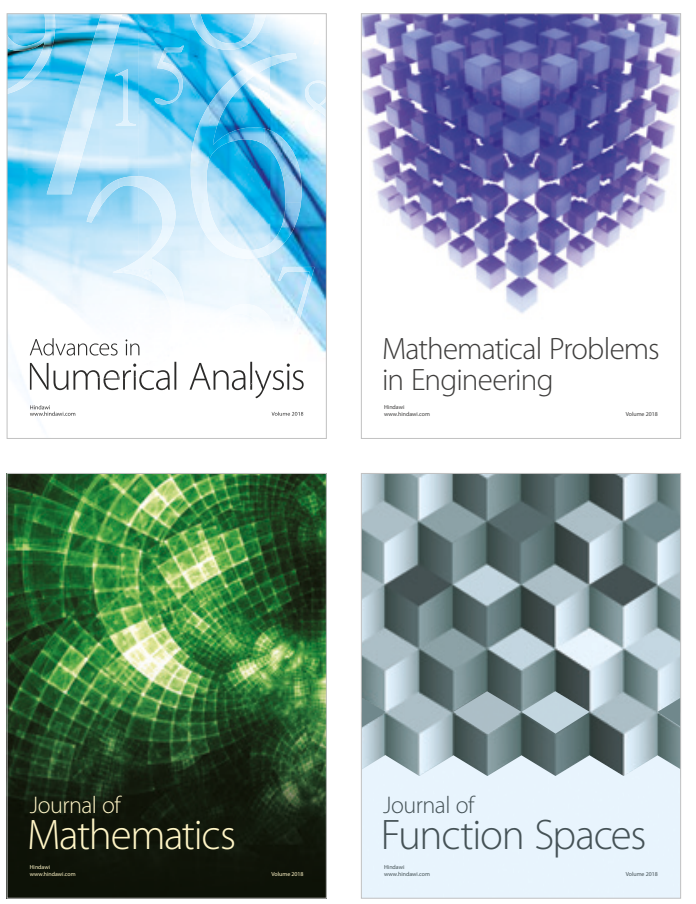

Mathematical Problems in Engineering

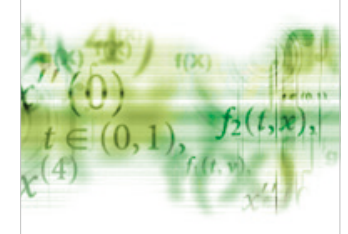

International Journal of

Differential Equations

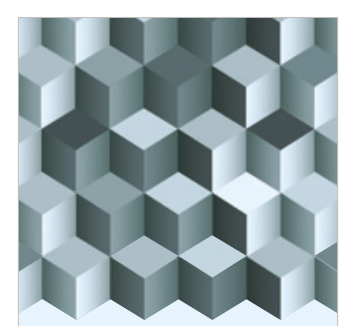

Journal of

Function Spaces
The Scientific

World Journal

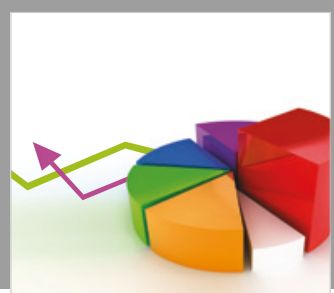

Journal of

Probability and Statistics
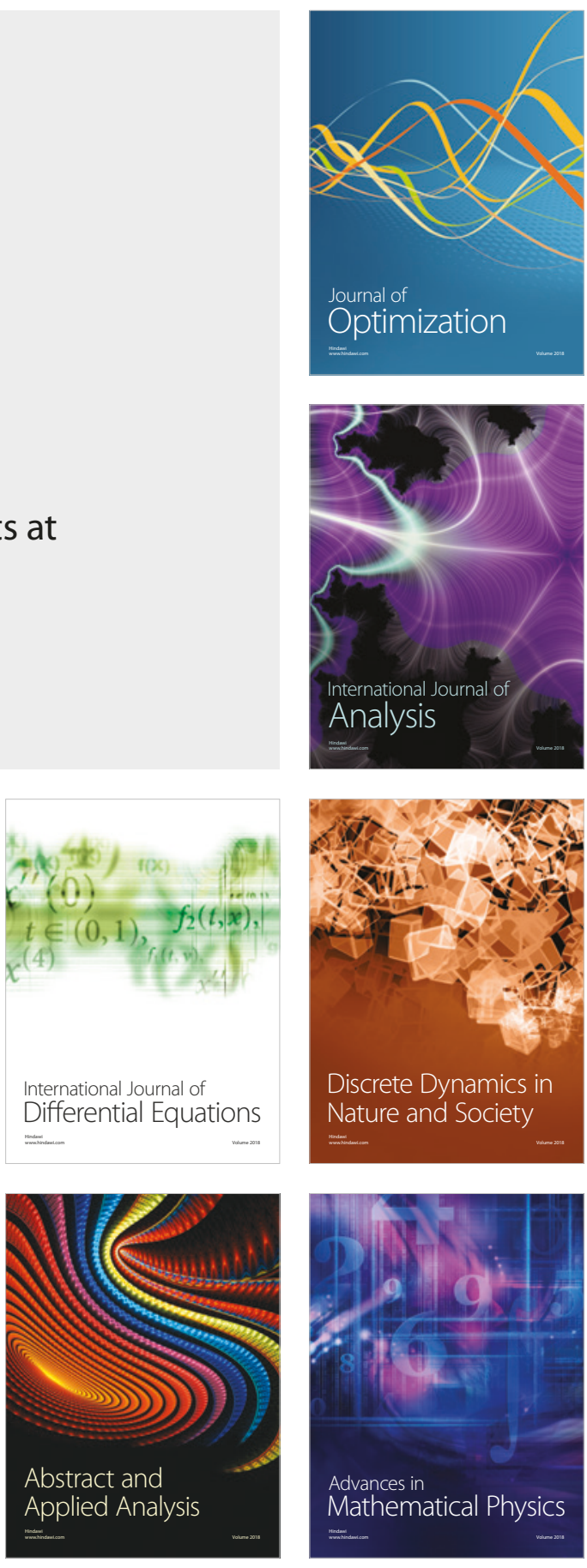